\title{
ON THE CHARACTERS OF THE PROLONGED DIFFERENTIAL SYSTEM
}

\author{
ISAO HAYASHI
}

\section{§ 1. Introduction}

Let $M=M(D, \pi)$ be a (real analytic) fibered manifold of dimension $n$ over a manifold $D$ of dimension $p$, with projection $\pi$. We denote by $M^{\prime}(D, \alpha)$ the prolonged fibered manifold of $M(D, \pi)$. Every point of $M^{\prime}$ is a $p$-dimensional contact element of $M$, and a $p$-dimensional contact element $X$ of $M$ belongs to $M^{\prime}$ if and only if $\pi_{*} X=T_{\pi(x)}(D)$, where $x$ is the origin of $X$. We write $x=$ $\beta(X)$ and $\alpha=\pi^{\circ} \beta$. We also denote by $M^{\prime \prime}\left(D, \alpha^{\prime}\right)$ the prolonged fibered manifold of $M^{\prime}(D, \alpha)$, where $\alpha^{\prime}=\alpha \circ \beta^{\prime}$ and $\beta^{\prime}$ is the projection of each $X^{\prime} \in M^{\prime \prime}$ to its origin in $M^{\prime}$.

Let $\mathfrak{U}$ be a (homogeneous and $d$-closed real analytic) differential system on the fibered manifold $M(D, \pi)$. Then, on the fibered manifold $M^{\prime}(D, \alpha)$, the prolonged differential system of $\mathfrak{A}$ is well defined. We denote it by $\mathfrak{A}$ '. Let $X^{\prime} \in M^{\prime \prime}$ and $X \in M^{\prime}$ be integral elements of $\mathfrak{H}^{\prime}$ and $\mathfrak{A}$ respectively. We denote their characters by $s_{k}^{\prime}\left(X^{\prime}\right)$ and $s_{k}(X) k=0,1, \ldots, p$. The following theorem was first proved by E. Cartan [1] in the special case and later by Matsushima [3] in the general case.

Theorem. If $X^{\prime}$ is an integral element of $\mathfrak{X}^{\prime}$ such that $X=\beta^{\prime}\left(X^{\prime}\right)$ is an ordinary integral element of $\mathfrak{U}$, then $X^{\prime}$ is also an ordinary integral element of $\mathfrak{U}^{\prime}$ and the characters satisfy the following relations

$$
s_{k}^{\prime}\left(X^{\prime}\right)=\sum_{j=k}^{p} s_{j}(X), \quad k=1,2, \ldots, p-1 .
$$

In this note we investigate the case where it is not assumed that $X$ is ordinary. In this case we have the inequalities (2) instead of (1). In fact, we can prove the following theorem.

ThEOREM. Let $X^{\prime}$ be an arbitrary integral element of $\mathfrak{I}^{\prime}$ and let $X$ be its origin.

Received November 30, 1965. 
Then the following inequaities hold:

$$
s_{k}^{\prime}\left(X^{\prime}\right) \leqq \sum_{j=k}^{p} s_{j}(X), \quad k=0,1, \ldots, p .
$$

Further, if the sets of 0 -forms of $\mathfrak{H}^{\prime}$ and $\mathfrak{Q}$ are regular at $X$ and $x=\beta(X)$ respectively, then the element $X$ is an ordinary integral element of $\mathfrak{A}$ if and only if (1) hold for all $k=0,1, \ldots, p$.

In $\S 2$, we state some definitions and lemmas and in $\S 3$ give a proof of our theorem.

\section{§ 2. Definitions and Lemmas}

Let $X \in M^{\prime}$ be an integral element of $\mathfrak{A}$, and $E^{k}$ be a $k$-dimensional element in $X$. We denote by $t\left(E^{k}\right)$ the rank of the polar system for $E^{k}$, and by $t_{k}(X)$ the maximum value of $t\left(E^{k}\right)$ for arbitrary $E^{k} \subset X$. Then, as is well known, there is a flag $\beta(X)=E^{0} \subset E^{1} \subset \cdots \subset E^{p-1} \subset X$, such that $t_{k}(X)=t\left(E^{k}\right)$ for $k=0,1, \ldots, p-1$. Such a flag $\left\{E^{k}\right\}$ is called a non-singular flag in $X$. Let $x=\beta(X), \bar{x}=\pi(x)$ and $\pi_{*} E^{k}=\bar{E}^{k}$. Then we have a flag $\left\{\bar{E}^{k}\right\}$ in $T_{\bar{x}}(D)$. Conversely, for any flag $\left\{\bar{E}^{k}\right\}$ in $T_{\bar{x}}(D)$, there is a unique flag $\left\{E^{k}\right\}$ in the given $X$, which is over the flag $\left\{\bar{E}^{k}\right\}$. An ordered coordinate system $\left(x^{1}, \ldots, x^{p}\right)$ at $\bar{x}$ in $D$ is called non-singular for $X$, if and only if the flag in $X$, which is over the flag $\left\{\left(\bar{\partial}_{1}, \ldots, \bar{\partial}_{k}\right)\right\}$, is non-singular (We denote by $\left(\bar{\partial}_{1}, \ldots, \bar{\partial}_{k}\right)$ the element spanned by vectors $\left.\bar{\partial}_{j}=\left(\partial / \partial x^{j}\right)_{\bar{x}}, j=1, \ldots, k\right)$.

Let $\left(x^{i}\right)$ be a non-singular coordinate system at $\bar{x}=\alpha(X)$ for an integral element $X$, such that $x^{i}(\bar{x})=0$. Then it is easily seen that there is an open and dense subset, say $G$, of $G L(p)$, such that any coordinate system $\left(y^{i}\right)$ at $\bar{x}$, defined by $y^{i}=\sum_{j} \alpha_{j}^{i} x^{i}$, is non-singular for $X$ if $\left(\alpha_{j}^{i}\right) \in G$. By this remark, we can easily verify the following lemma.

Lemma 1. Let $M_{1}\left(D, \pi_{1}\right)$ and $M_{2}\left(D, \pi_{2}\right)$ be fibered manifolds over the same manifold $D$, and let $\mathfrak{H}_{1}$ and $\mathfrak{H}_{2}$ be differential systems on $M_{1}\left(D, \pi_{1}\right)$ and $M_{2}(D$, $\pi_{2}$ ) respectively. If $X_{1}$ and $X_{2}$ are integral elements of $\mathfrak{H}_{1}$ and $\mathfrak{H}_{2}$ respectively such that their origins are over the same point $\bar{x}$ of $D$, then there exists $a$ coordinate system $\left(x^{i}\right)$ at $\bar{x}$, which is non-singular for both $X_{1}$ and $X_{2}$.

For any integral element $X$ of $\mathfrak{A}$, we denote by $t_{-1}(X)$ the rank of $d \mathfrak{T}_{\beta(X)}^{(o)}$, where $\mathfrak{I}^{(0)}$ is the set of 0 -forms of $\mathfrak{A}$. We define the characters $s_{k}(k=0$, 
$1, \ldots, p)$ by $s_{k}(X)=t_{k}(X)-t_{k-1}(X)$, for $k=0, \ldots, p-1$, and by $s_{p}(X)=n-$ $p-t_{p-1}(X)$. Our $s_{k}$ are the same as the ones in [3] except $s_{0}$.

Let $\{f\}$ be a set of (real analytic) functions defined on a neighborhood of a point $a$ in a manifold and $f(a)=0$ for every $f \in\{f\}$. We say that $\{f\}$ is regular at the point $a$, if there exist a subset $\left\{f_{1}, \ldots, f_{m}\right\}$ of $\{f\}$ and a neighborhood $V$ of $a$, such that (i) $\left(d f_{1}\right)_{a}, \ldots,\left(d f_{m}\right)_{a}$ are linearly independent, and (ii) if $p \in V$ and $f_{i}(p)=0, i=1, \ldots, m$, then $f(p)=0$ for any $f \in\{f\}$.

An integral element $X$ of the differential system $\mathfrak{A}$ on the fibered manifold $M(D, \pi)$ is called ordinary if and only if the following conditions (i) and (ii) are satisfied:

(i) $\mathfrak{H}^{(0)}$ and $\mathfrak{U}^{(0)}$ are regular at $\beta(X)$ and $X$ respectively,

(ii) the dimension of the submanifold of $M^{\prime}$, defined by the set of equations $\mathfrak{U}^{(\circ)}=0$ in a neighborhood of $X$, is $n^{\prime}-t_{-1}(X)+\sum_{k=1}^{\prime \prime} k s_{k}(X)$, where $n^{\prime}=\operatorname{dim} M^{\prime}$ $=n+p(n-p)$.

For later use, we state the following

Lemma 2. Let $V$ be a finite dimensional vector space and $V=V_{0}+V_{1}+\cdots$ $+V_{p+1}$ be a direct sum decomposition of $V$. Denote by $i_{k_{1}, \ldots, k_{q}}$ the identity map of $V_{k_{1}}+\cdots+V_{k_{q}}$ into $V$ and suppose that a family of sets of linear functionals $\left\{\Psi^{(k)} \mid k=0,1, \ldots, p\right\}$ on $V$ is given and each set of linear functionals $\Psi^{(k)}$ satisfies the condition $i_{k+1, k+2, \ldots, p+1}^{*} \Psi^{(k)}=\{0\}$. Then there exists a set of non-negative integers $\left\{\delta_{0}, \delta_{1}, \ldots, \delta_{p-1}\right\}$, such that

$$
\operatorname{rank}\left(i_{k, k+1}^{*}, \ldots, p+1\left(\bigcup_{l=0}^{p} \Psi^{(l)}\right)\right)=\sum_{j=k-1}^{p-1} t_{j}+\sum_{j=k}^{p-1} \delta_{j}, k=0,1, \ldots, p,
$$

where $t_{k-1}=\operatorname{rank}\left(i_{k}^{*} \Psi^{(k)}\right)$.

This lemma is easily verified by applying the following Lemma 3 repeatedly.

Lemma 3. Let $V=V_{0}+V_{1}$ be a direct sum decomposition of a finite dimensional vector space $V$, and $\Psi_{0}$ and $\Psi_{1}$ be sets of linear functionals on $V$ such that $i_{1}^{*} \Psi_{0}=$ $\{0\}$, rank $\left(i_{0}^{*} \Psi_{0}\right)=t_{0}$ and $\operatorname{rank}\left(i_{1}^{*} \Psi_{1}\right)=t_{1}$. Then, for some $\delta \geqq 0, \operatorname{rank}\left(\Psi_{0} \cup \Psi_{1}\right)=$ $t_{0}+t_{1}+\delta$.

This lemma is obvious.

\section{§3. Proof of Theorem}

Let $X^{\prime}$ be an integral element of $\mathfrak{A}^{\prime}, \beta^{\prime}\left(X^{\prime}\right)=X$ and $\beta(X)=x$. By Lemma 
1 , we can find a coordinate system $\left(x^{i}\right)$ at $\pi(x)$ in $D$, which is non-singular for both $X^{\prime}$ and $\dot{X}$. Since $M(D, \pi)$ is a fibered manifold, there is a coordinate system $\left(x^{i} \circ \pi, x^{\lambda}\right), i=1, \ldots, p ; \lambda=p+1, \ldots, n$, defined on a neighborhood $U$ of $x \in M$. We shall use the notation $x^{i}$ instead of $x^{i} \circ \pi$, and adopt such conventions in the sequel. Let $U^{\prime}=\beta^{-1}(U)$. The coordinate system $\left(x^{i}, x^{\lambda}\right)$ on $U$ introduces the canonical coordinate system $\left(x^{i}, x^{\lambda}, i_{i}^{\lambda}\right)$ on $U^{\prime}$.

We write $\partial_{i}=\partial / \partial x^{i}, \partial_{\lambda}=\partial / \partial x^{\lambda}, \partial_{\lambda}^{i}=\partial / \partial l_{i}^{\lambda}$ and put $e_{i}=\partial_{i}+\sum_{\lambda} l_{i}^{\lambda} \partial_{\lambda}, e_{\lambda}=\partial_{\lambda}$. These are vector fields on $U^{\prime}$, and every $X \in U^{\prime}$ is spanned by $\beta_{*}\left(e_{i}\right)_{X}, i=1$, ...,p.

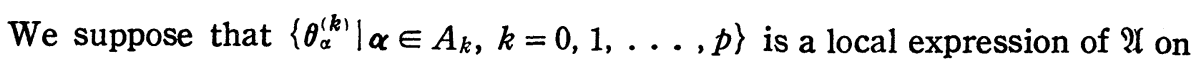
$U$ where $\left\{\theta_{\alpha}^{(k)} \mid \alpha \in A_{k}\right\}$ is the set of $k$-forms and $\left\{d \theta_{\alpha}^{(k)} \mid \alpha \in A_{k}\right\} \subset\left\{\theta_{\alpha}^{(k+1)} \mid \alpha \in A_{k+1}\right\}$. ( $A_{k}$ are sets of indices). For vector fields $e_{j_{1}}, \ldots, e_{j_{k}}\left(1 \leqq j_{1}, \ldots, j_{k} \leqq n\right)$ defined above, $\left\langle\beta^{*} \theta_{\alpha}^{(k)}, e_{j_{1}} \wedge \cdots \wedge e_{j_{b}}\right\rangle$ is a function on $U^{\prime}$. We denote it by $H_{\alpha j_{1} \cdots j_{k}}^{(k)} \quad$ (In particular, $\left.H_{\alpha}^{(\rho)}=\beta^{*} \theta_{\alpha}^{(\rho)}\right)$. We consider a set

$$
\Theta=\left\{H_{\alpha i_{1} \cdots i_{k}}^{(k)} \mid 1 \leqq i_{1}<\cdots<i_{k} \leqq p, \alpha \in A_{k}, k=0,1, \ldots, p\right\},
$$

of functions on $U^{\prime}$ and a set of 1 -forms $\varphi^{\lambda}=d x^{\lambda}-\sum_{i} l_{i}^{\lambda} d x^{i},(\lambda=p+1, \ldots, n)$ on $U^{\prime}$. Then

$$
\Theta \cup d \Theta \cup\left\{\varphi^{\lambda}\right\} \cup\left\{d \varphi^{\lambda}\right\}
$$

is a local expression of $\mathfrak{H}^{\prime}$ on $U^{\prime}$.

Now we write, for the given points $X$ and $x=\beta(X), e_{j}(x)=\beta_{*}\left(e_{j}\right)_{x}$ and $\varphi_{x}^{\lambda}=\left(d x^{\lambda}\right)_{x}-\sum_{i} l_{i}^{\lambda}(X)\left(d x^{i}\right)_{x}$. Then $\left(e_{i}(x), e_{\lambda}(x)\right)$ is a basis of the vector space $T_{x}(M)$, and $\left(\left(d x^{i}\right)_{x}, \varphi_{x}^{\lambda}\right)$ is the dual basis. Hence we have

$$
\begin{aligned}
& \left(\theta_{\alpha}^{(l)}\right)_{x} \mathrm{~L}\left(e_{i_{1}}(x) \wedge \cdots \wedge e_{i_{l-1}}(x)\right)=\sum_{i}<\left(\theta_{\alpha}^{(l)}\right)_{x}, e_{i_{1}}(x) \wedge \cdots \wedge e_{i_{l-1}}(x) \\
\wedge & e_{i}(x)>\left(d x^{i}\right)_{x}+\sum_{\lambda}<\left(\theta_{\alpha}^{(l)}\right)_{x}, e_{i_{1}}(x) \wedge \cdots \wedge e_{i_{l-1}}(x) \wedge e_{\lambda}(x)>\varphi_{x}^{\lambda} \\
= & \sum_{\lambda} H_{\alpha i_{1} \cdots j_{l-l^{\lambda}}}^{(l)}(X) \varphi_{z}^{\lambda} .
\end{aligned}
$$

Therefore

(4) $\Phi^{(k-1)}=\left\{\sum H_{\alpha i_{1} \cdots i_{l-1} \lambda}^{(l)}(X) \varphi_{x}^{\lambda} \mid 1 \leqq i_{1}<\cdots<i_{l-1} \leqq k-1, \alpha \in A_{l}, l=1, \ldots, k\right\}$ is the polar system for the $(k-1)$-dimensional element $\left(e_{1}(x), \ldots, e_{k-1}(x)\right)$, for $k=1, \ldots, p$. In addition, putting

$$
\emptyset^{(-1)}=\left\{\left(d \theta_{\alpha}^{(\circ)}\right)_{x} \mid \alpha \in A_{0}\right\}
$$


we have $\operatorname{rank}\left(\Phi^{(k-1)}\right)=t_{k-1}(X)$ for $k=0,1, \ldots, p$.

We now proceed to examine the characters $s_{k}^{\prime}\left(X^{\prime}\right)$. We suppose that $X^{\prime}$ is spanned by $e_{1}^{\prime}, \ldots, e_{p}^{\prime}$, where

$$
e_{i}^{\prime}=\left(\partial_{i}\right)_{X}+\sum_{\Lambda} t_{i}^{\lambda}\left(\partial_{\lambda}\right)_{x}+\sum_{\Lambda \jmath} t_{i j}^{\lambda}\left(\partial_{\lambda}^{i}\right)_{x}, i=1, \ldots, p .
$$

Since $X^{\prime}$ is the integral element of $\mathfrak{F}^{\prime}$ with the origin $X$, we have $t_{i}^{\lambda}=l_{i}^{\lambda}(X)$, $t_{i j}^{\lambda}=t_{j i}^{\lambda}$ and $e_{i}^{\prime}(H)=0$ for any $H \in \Theta$. If we set $\psi^{\lambda}=\left(d x^{\lambda}\right)_{X}-\sum_{i} t_{i}^{\lambda}\left(d x^{i}\right)_{X}$ and $\psi_{i}^{\lambda}=\left(d l_{i}^{\lambda}\right)_{X}-\sum_{j} t_{i j}^{\lambda}\left(d x^{j}\right)_{x}$, we can see that the polar system for the $(k-1)$ dimensional element $\left(e_{1}^{\prime}, \ldots, e_{k-1}^{\prime}\right)$ is given as follows:

$$
\begin{aligned}
\Phi^{\prime(k-1)} & =(d \Theta)_{X} \cup\left\{\phi^{\lambda} \mid \lambda=p+1, \ldots, n\right\} \cup\left\{\psi_{i}^{\lambda} \mid i=1, \ldots, k-1 ;\right. \\
\lambda & =p+1, \ldots, n\} .
\end{aligned}
$$

We have $(d \Theta)_{x}=\bigcup_{k=0}^{p} \Psi^{(k)}$ by setting

$$
\Psi^{(0)}=\left\{\left(d H_{\alpha}^{(0)}\right)_{x} \mid \alpha \in A_{\circ}\right\}
$$

and

$$
\Psi^{(k)}=\left\{\left(d H_{\alpha i_{1} \cdots i_{l-1} k}^{(l)}\right)_{x} \mid 1 \leqq i_{1}<\cdots<i_{l-1} \leqq k-1, \alpha \in A_{l}, l=1, \ldots, k\right\} .
$$

Since $\left(\left(d x^{i}\right)_{x}, \psi^{\lambda}, \psi_{i}^{\lambda}\right)$ is the dual basis of $\left(e_{i}^{\prime},\left(\partial_{\lambda}\right)_{X},\left(\partial_{\lambda}^{i}\right)_{X}\right)$ in $T_{X}\left(M^{\prime}\right)$, we have $(d H)_{X}=\sum_{\lambda}\left(\partial_{\lambda}\right)_{X}(H) \psi^{\lambda}+\sum_{\lambda \iota}\left(\partial_{\lambda}^{i}\right)_{X}(H) \psi_{i}^{\lambda}$ for any $H \in \Theta$.

Therefore we have

$$
\left(d H_{\alpha i_{1} \cdots i_{l-1} k}^{\prime \prime}\right)_{X} \equiv \sum_{\lambda} H_{\alpha i_{1} \cdots i_{l-1} \lambda}^{(l)}(X) \phi_{k}^{\lambda} \quad\left(\bmod \phi^{\lambda}, \phi_{i_{1}}^{\lambda}, \ldots, \varphi_{i l-1}^{\lambda}\right), l \geqq 1 .
$$

We consider the direct sum decomposition $T_{x}\left(M^{\prime}\right)=V_{0}+V_{1}+\cdots+V_{p+1}$, where $V_{0}=\left(\left(\partial_{p+1}\right)_{X}, \ldots,\left(\partial_{n}\right)_{X}\right), V_{k}=\left(\left(\partial_{p+1}^{k}\right)_{x}, \ldots,\left(\partial_{n}^{k}\right)_{X}\right), k=1, \ldots, p$, and $V_{p+1}=X^{\prime}$. We can apply Lemma 2 to our vector space $T_{X}\left(M^{\prime}\right)$ and the family of sets of linear functionals $\left\{\varphi^{(k)}\right\}$ defined by (7) and (8). In fact, we can see $i_{k+1, \ldots, p+1}^{*} \Psi^{(k)}=\{0\}$ for $k=0,1, \ldots, p$, by $(7),(8)$ and (9). Further we have, $\operatorname{rank}\left(i_{k}^{*} \Psi^{(k)}\right)=t_{k-1}(X)$ for $k=1, \ldots, p$ by $(4),(8),(9)$ and for $k=0$ by (5), (7). Therefore, by Lemma 2, there exists a set of non-negative integers $\left\{\delta_{0}, \ldots, \delta_{p-1}\right\}$ such that

$$
\begin{aligned}
& t_{-1}^{\prime}\left(X^{\prime}\right)=\operatorname{rank}\left((d \Theta)_{X}\right)=\sum_{j=-1}^{n-1} t_{j}(X)+\sum_{j=0}^{p-1} \delta_{j}, \\
& t_{k-1}\left(X^{\prime}\right)=\operatorname{rank}\left({\Phi^{\prime}}^{(k-1)}\right)=k(n-p)+\operatorname{rank}\left(i_{k, \ldots, p+1}^{*}(d \Theta)_{X}\right)
\end{aligned}
$$




$$
=k(n-p)+\sum_{j=k-1}^{p-1} t_{j}(X)+\sum_{j=k}^{p-1} \delta_{j} \text { for } k=1, \ldots, p-1
$$

and $t_{p-1}^{\prime}\left(X^{\prime}\right)=p(n-p)+t_{p-1}(X)$.

Since by definition, $s_{k}^{\prime}\left(X^{\prime}\right)=t_{k}^{\prime}\left(X^{\prime}\right)-t_{k-1}^{\prime}\left(X^{\prime}\right)$ for $k=0,1, \ldots, p-1$ and $s_{p}^{\prime}\left(X^{\prime}\right)=n^{\prime}-p-t_{p-1}^{\prime}\left(X^{\prime}\right)$, we obtain

$$
\left\{\begin{array}{l}
s_{k}^{\prime}\left(X^{\prime}\right)=\sum_{j=k}^{p} s_{j}(X)-\delta_{k}, k=0,1, \ldots, p-1, \\
s_{p}^{\prime}\left(X^{\prime}\right)=s_{p}(X) .
\end{array}\right.
$$

Since $\delta_{k} \geqq 0$, the inequalities (2) in the theorem are verified.

The latter half of the theorem also follows from the above computations. Since the set of functions $\Theta$ is regular at $X$ by the assumption, the submanifold of $M^{\prime}$, defined by $\Theta=0$ in a neighborhood of the point $X$, is of dimension $n^{\prime}$ $-t_{-1}^{\prime}\left(X^{\prime}\right)=n-t_{-1}(X)+\sum_{k=1}^{p} k s_{k}(X)-\sum_{k=0}^{p-1} \delta_{k}$. Therefore $X$ is ordinary if and only if $\sum_{k=0}^{p-1} \delta_{k}=0$. We can see by (10) that this condition is equivalent to the equalities (1) for all $k=0,1, \ldots, p$. Thus the proof is complete.

\section{REFERENCES}

[1] E. Cartan, Sur la structure des groupes infinis de transformations (1904). Oeuvres completes. Partie 2. vol. 2, pp 571-624.

[2] E. Cartan, Les systemes differentiels exterieurs et leurs applications geometriques. Paris. (1945).

[3] Y. Matsushima, On a theorem concerning the prolongation of a differential system. Nagoya. Math. J. 6 (1953), pp 1-16.

\section{Mathematical Institute}

Nagoya University 